\title{
Geostatistical analysis of surface morphology and its relationship with hydro-mechanical properties of natural fractures
}

\author{
W. Kosasih \& V. Rasouli \\ Curtin University, Department of Petroleum Engineering, Australia
}

\begin{abstract}
The morphological properties of rock fractures are intimately linked with its hydro-mechanical behaviours. In this paper, the relationship between the two properties for a real rock like fracture is studied using geostatistical analysis techniques. Surface morphology of the fracture is modelled through variograms for different directions to determine the anisotropy extent of the fracture's roughness. The results are then compared to the results of a fluid flow simulation undertaken for a separate study to determine whether any correlation exists between the two properties. Observations suggest a clear correlation between directional dependence of the asperity elevations of a fracture surface and fluid pressure drop. Thus, geostatistical modelling of surface morphology can potentially be used to predict and understand the hydro-mechanical properties of a fracture which can prove to be valuable in real applications such as minimising the negative impacts of fractures to hydrocarbon production.

Keywords: fracture morphology, fluid flow, geostatistical analysis, variogram modelling, anisotropy.
\end{abstract}

\section{Introduction}

The analysis of fluids flowing through fractures in a reservoir requires a different approach to standard models of fluid flow in porous media. Both natural and man-made fractures commonly exist within petroleum reservoirs [1] and consequently, petroleum production is often influenced by the fluid flow behaviour inside these fractures. With this premise in mind, an understanding of 
how fluids flow through a fractured surface with relation to surface morphology can aid in minimizing any negative impacts to production that may be induced by rock fractures within the reservoir.

Numerous investigations of fluid flow along rough surfaces have been performed in the past. Giacomoni et al. [2] studied the flow anisotropy within a natural joint subjected to mechanical shearing where surface roughness was taken into account statistically through the definition of a coefficient that reduced permeability. A study by Witherspoon et al. [1] examined fluid flow in fractured rock masses by modelling rock fractures as either a collection of voids or an array of asperities. Stochastics and numerical simulations that incorporated fracture surface roughness were used by Dicman et al. [3] to describe flow on a rough fracture surface. A numerical code was proposed by Kamath et al. [4] to calculate single phase fluid flow in naturally fractured reservoirs. All of these studies suggest that fracture surface roughness acts to hinder the flow of fluids travelling along its surface.

Previous studies have also investigated the relationship of morphological anisotropy and the hydro-mechanical properties of fracture surfaces through the use of geostatistical techniques. Geostatistical descriptions of fracture surface topography were used by Piggott and Elsworth [5] to describe the mechanical and hydraulic behaviour of rock fractures subjected to shear loading. The conclusion was made that surface roughness influences fracture fluid flow properties and that anisotropy in surface roughness characteristics will lead to anisotropy in the hydro-mechanical properties of the fracture. As part of a study by Roko et al. [6], the anisotropy characteristics of joint surface morphology were modelled using variogram analysis. It was described how directions of low sill represent the global flow path of the joint surface and that the direction with high sill provides higher resistance that will act to hinder fluid flow.

The geostatistical modelling technique discussed has gained popularity in recent years within the petroleum industry for its ability to describe the spatial dependence of reservoir attributes, a factor that is ignored in conventional statistics. By taking advantage of the fact that for most variables, sample measurements at two nearby locations tend to be more similar in value than sample measurements taken from two distant locations [7], a sense of the variable's directional dependence can be established through the use of variogram analysis. This information can subsequently be used as data feed for interpolation methods such as Kriging to generate optimal interpolations at points on the field with lack of sampling data [7].

This study will focus on the application of these geostatistical techniques in determining the relationship between surface morphology and the hydromechanical properties of a real rock like fracture that was originally made for shear tests for a different experiment. The results of the geostatistical model is compared to the results of a fluid flow simulation carried out for a separate study to see whether any correlations between the fluid pressure drop and the asperity elevation anisotropy of a rock fracture surface can be observed. 


\section{Geostatistical modelling}

Geostatistical modelling functions on the assumption that for most variables, sample measurements at two nearby locations tend to be more similar in value than sample measurements taken from two distant locations. The differences in the values of these sample measurements is termed the semivariance and is plotted against the distance between the two points measured, termed the lag, to create the chart known as the variogram [8]. The variogram illustrates the semivariance of a variable as a function of separation distance along a direction in a given space. The analysis of anisotropy in roughness of fractured surfaces using geostatistical modelling is the objective of this study. The variogram analysis at different directions will be used for this purpose.

\subsection{Variogram models}

The graph of semivariance against separation distance typically yields a curve similar to Figure 1. Common theoretical variogram models to fit these curves

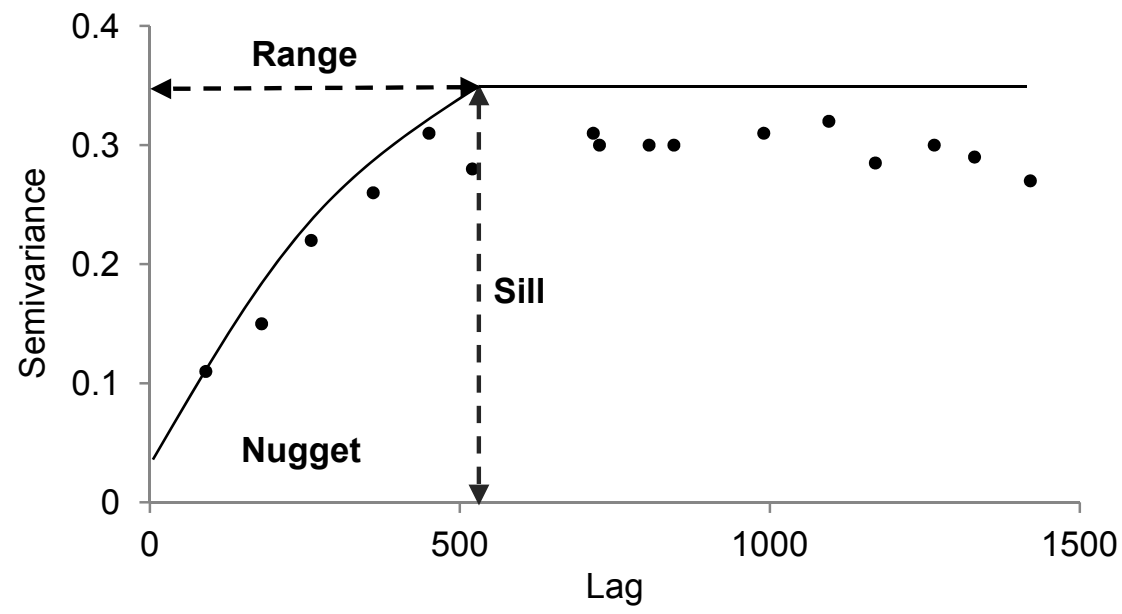

Figure 1: A typical variogram, displaying its sill, range and the nugget effect.

include linear, spherical, exponential and Gaussian. These models can be divided into models with sill (spherical, exponential and Gaussian) and models without sill (linear) [6]. For the purposes of this study, the spherical model shall be used and its equation is given as:

$$
\begin{gathered}
\gamma(h)=C_{0}+C\left[1.5\left(\frac{h}{A_{0}}\right)-0.5\left(\frac{h}{A_{0}}\right)^{3}\right] \quad \text { for } h \leq A_{0} \\
\gamma(h)=C_{0}+C \quad \text { for } h>A_{0}
\end{gathered}
$$

where $\gamma(h), h, C_{0}, C, A_{0}$ denotes semivariance, lag distance interval, nugget variance, structural variance and range, respectively. 


\subsection{Variogram parameters}

The variogram is characterised by a couple of different parameters. If the separation distance between two sampling measurements of a variable is zero, the two sampling points effectively lie on the same point in space and the corresponding semivariance should theoretically measure a zero value [8]. Any variation from this is termed the nugget effect and manifests itself in duplicate data analysis. The nugget effect can be seen in Figure 1 as the curve fails to pass through the origin.

The range of a variogram represents the maximum distance of spatial autocorrelation and is represented on the variogram curve as the lag value where the sill begins, as shown in Figure 1.

The extent of the variable anisotropy can be characterised through the value known as the sill of the variogram [9]. As separation distance between sampling points increase, so too does the difference between the measured values of the variables. The semivariance will increase until a point where it reaches a limit and plateaus to a maximum value known as the sill, suggesting that there is now minimal dependence with respect to the property value between the two points measured. Depending on the context, the sill can refer to the full value of the semivariance at this point or it can refer to the value of semivariance at this point less the value of the nugget. Either way, the principle and meaning behind the sill remains the same.

Through these parameters, the variogram can be used to interpret the spatial dependence of the variable along a direction. Low values of sill indicate that the changes in the values of the variable along that particular direction is minimal and hence the variable is said to be directionally dependent along that direction. High values of sill on the other hand, indicate that the changes in the values of the variable along that particular direction is large and hence the variable is said to be directionally independent along said direction [9].

These sill values can be obtained for every direction of interest and from this the anisotropy extent of the variable for the area can be seen by plotting these values on a rose diagram, forming a chart known as the anisotropy ellipse. An example of an anisotropy ellipse of a variable in space is shown in Figure 2 where it is seen that the property is most anisotropic roughly along the $60^{\circ}$ direction and least anisotropic roughly along the $145^{\circ}$ direction.

\subsection{Geostatistics and data interpolation}

The use of statistics is often employed across a variety of disciplines as a tool for adapting to uncertainty and lack of data. Spatial relationship between data provides invaluable information for the crucial step of data interpolation into points without sampling data available when constructing a variogram. Using this data, optimal interpolations which generate the best unbiased estimate at each location is possible.

A number of algorithms exist for the interpolation of data into unobserved locations including inverse distance weighting, bilinear interpolation, nearestneighbour interpolation and Kriging [7]. The Kriging algorithm in particular, 


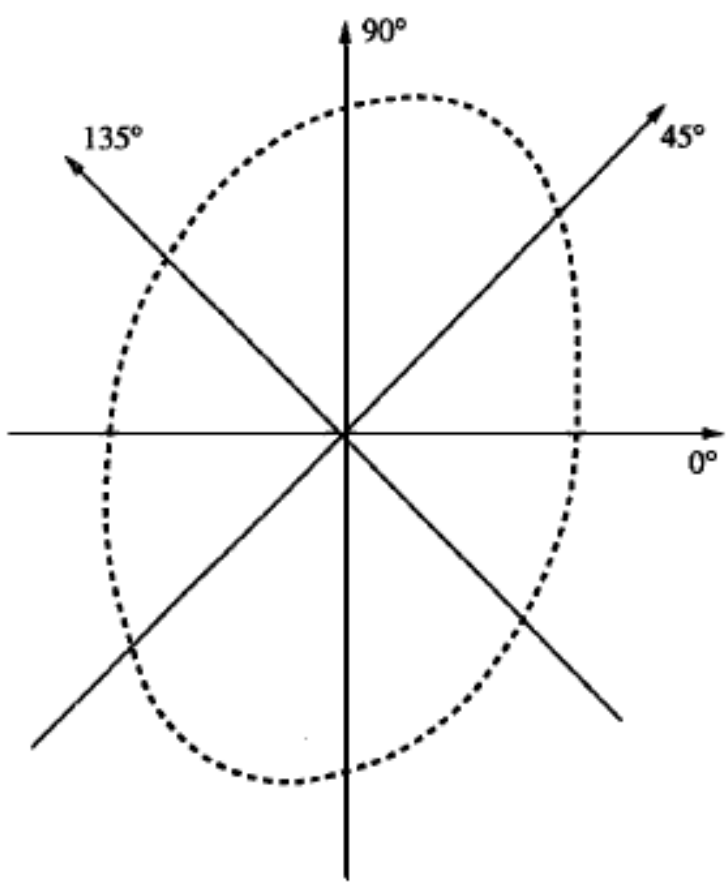

Figure 2: A typical anisotropy ellipse displaying sill values for a variable in space [6].

will be used in this study for not only the construction of the variograms but also to incorporate the geostatistical data produced in order to reconstruct a 3D model of the fracture surface used in this study (shown in Figure 4).

\section{Fracture morphology and hydro-mechanical properties}

The geometric morphology of a fracture has a profound impact on the behaviour of any fluids flowing through its surface. Consider a simple corrugated plane as shown in Figure 3. Any fluids flowing through along the $\mathrm{X}$ direction is expected to experience larger pressure drops than the same fluids travelling through the $\mathrm{Y}$ direction due to the fluids requiring more energy to overcome the asperities that acts to hinder the flow. Along the $\mathrm{Y}$ direction, the fluids flow through a smooth channel and should theoretically experience much less of a pressure drop compared to fluids flowing through the $\mathrm{X}$ direction. Fluid flow is directionally dependent as the surface roughness is anisotropic [10].

The same principles can be applied to the more complex real like fracture that is examined for this study. An artificial fracture sample was made out of mortar for shear studies in the lab. The sample is an accurate representation of what a real fracture would look like. The asperity elevations were extracted and digitized using digital photogrammetry. Figure 4 displays the 3D contour map of the sample surface. 


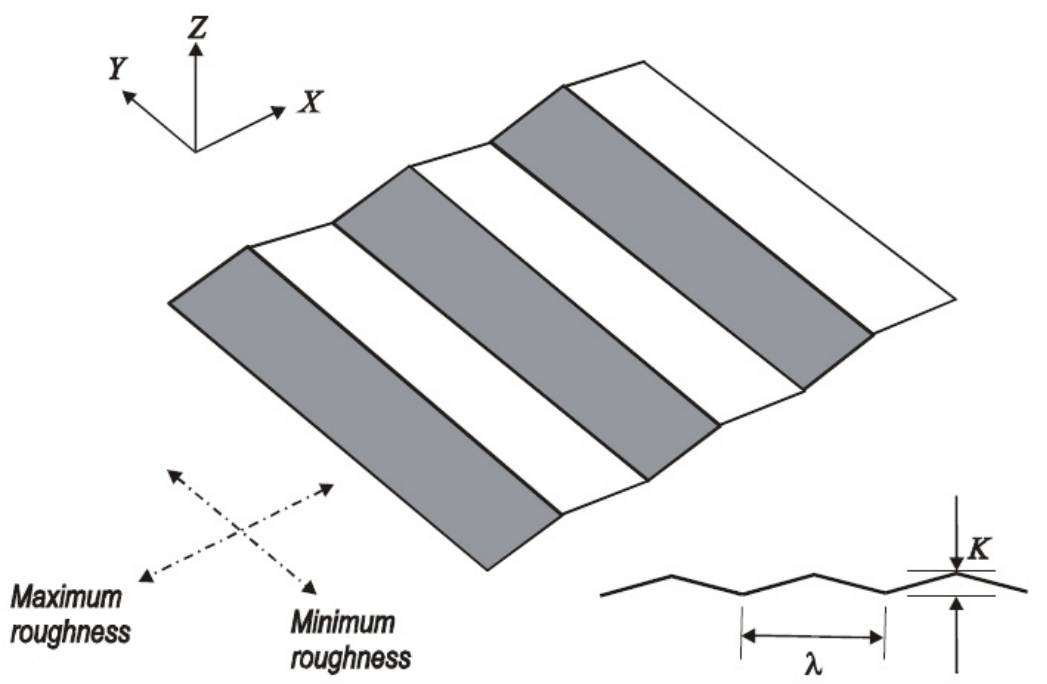

Figure 3: $\quad$ Model of a corrugated plane showing directions of maximum and minimum roughness [11].

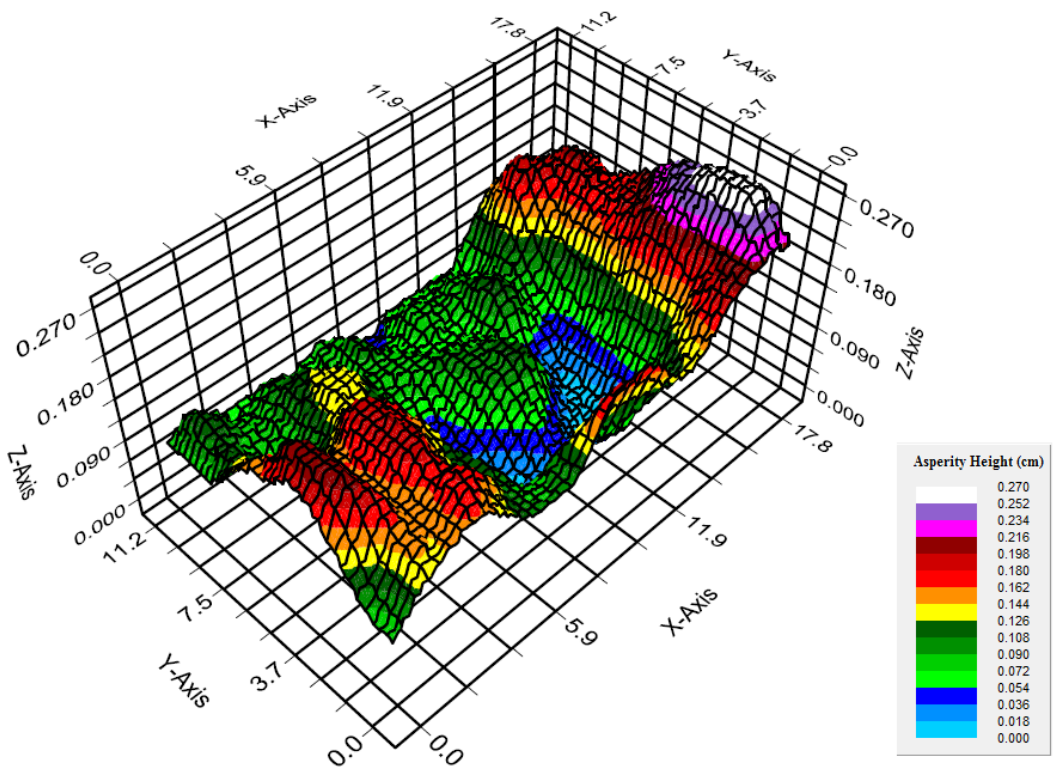

Figure 4: A fracture surface analysed in this study for its hydro-mechanical behaviour. 
The hydro-mechanical properties of this particular surface were examined by Hosseinian [10] for a separate study. Laminar fluid flow simulations were carried out using the FLUENT software for different directions and the results are shown in Figure 5. The chart illustrates how the fluid flow is directional dependent due to the anisotropy of the surface morphology. This study was initiated with the idea of exploring any correlation between surface roughness and hydro-mechanical properties of this surface. We employ the use of geostatistical methods for this purpose and the detailed discussions are given in the following section.

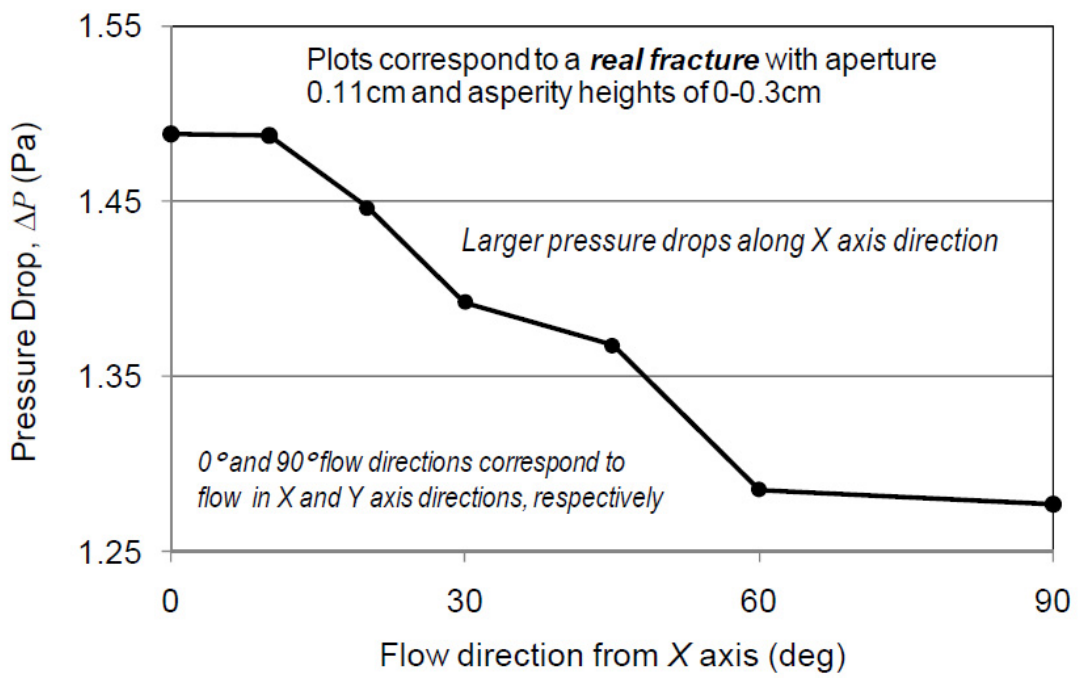

Figure 5: Pressure drop across the fracture surface shown in Figure 4 is directionally dependent [10].

\section{Geostatistical analysis}

In order to perform geostatistical analysis on the fracture surface, the data from the photogrammetry was taken and variograms were constructed of the asperity elevations using the methods discussed in section 2. Four variograms were charted for different directions at angles $0^{\circ}, 45^{\circ}, 90^{\circ}$ and $135^{\circ}$ measured clockwise from the north direction, respectively. The combination of these four azimuths provides a good representation of anisotropy over the whole surface of the mortar sample (the angles also correspond to directions of $180^{\circ}, 225^{\circ}, 275^{\circ}$ and $315^{\circ}$, respectively). These variograms are presented in Figure 6 and the anisotropy ellipse corresponding to the sill of variograms at different directions is shown in Figure 7, describing how surface morphology anisotropy is maximised at $90^{\circ}$ direction. 
Anisotropic Variogram $\left(0^{\circ}\right)$

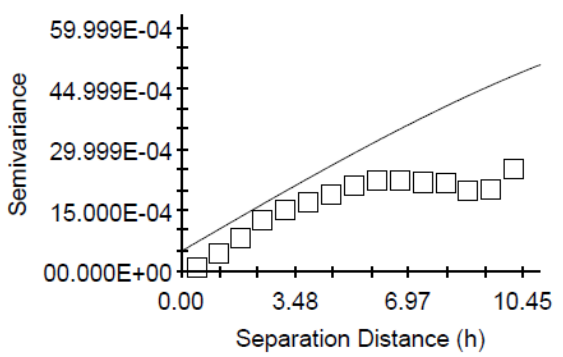

Anisotropic Variogram $\left(90^{\circ}\right)$

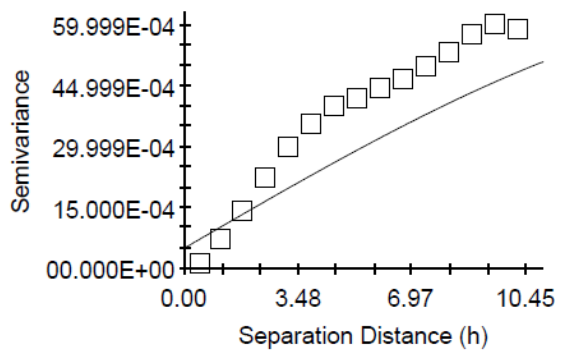

Anisotropic Variogram $\left(45^{\circ}\right)$

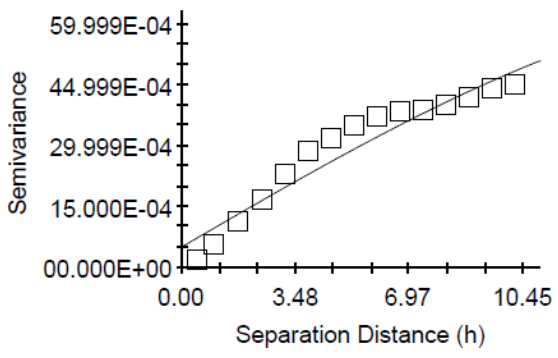

Anisotropic Variogram $\left(135^{\circ}\right)$

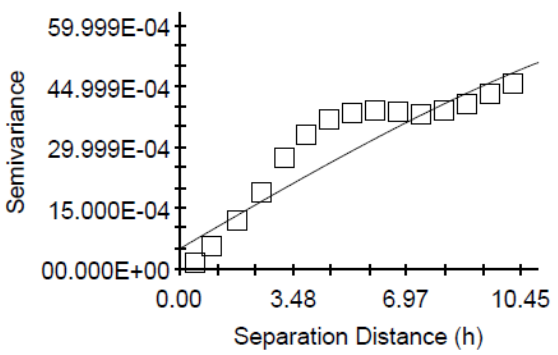

Figure 6: Variograms of the fracture surface elevation data corresponding to $0^{\circ}, 45^{\circ}, 90^{\circ}$ and $135^{\circ}$ directions.

These variograms can be used to interpret the spatial dependence of asperity elevation along a particular direction. From Figure 6, it can be seen that the value of the sill is substantially lower for the direction of zero degree compared to the other directions, suggesting that changes in asperity elevation along this direction is minimal (i.e. a relatively smooth direction). Asperity elevation is thus most directionally dependent along the direction of zero degree. In contrast, asperity elevation is seen to be least directionally dependent along the direction of $90^{\circ}$ with a higher sill value than the rest of the variograms, suggesting that changes in asperity elevation along this direction is high (i.e. a relatively rough direction).

With this data at hand, we can now compare the results of the geostatistical analysis of the rock surface anisotropy to the results of the FLUENT fluid flow simulations. Variograms indicate that sample asperity elevations are most anisotropic in the $\mathrm{X}$ direction $\left(90^{\circ}\right.$ variogram) and least anisotropic in the $\mathrm{Y}$ direction $\left(0^{\circ}\right.$ variogram). From Figure 5 it can be seen that fluids experience the highest pressure drop flowing through the $\mathrm{X}$ direction and least pressure drop when flowing in the $\mathrm{Y}$ direction.

The results of both studies are presented in Figure 8 for comparison purposes. The results shown in this figure clearly indicate correlations between the sill of 


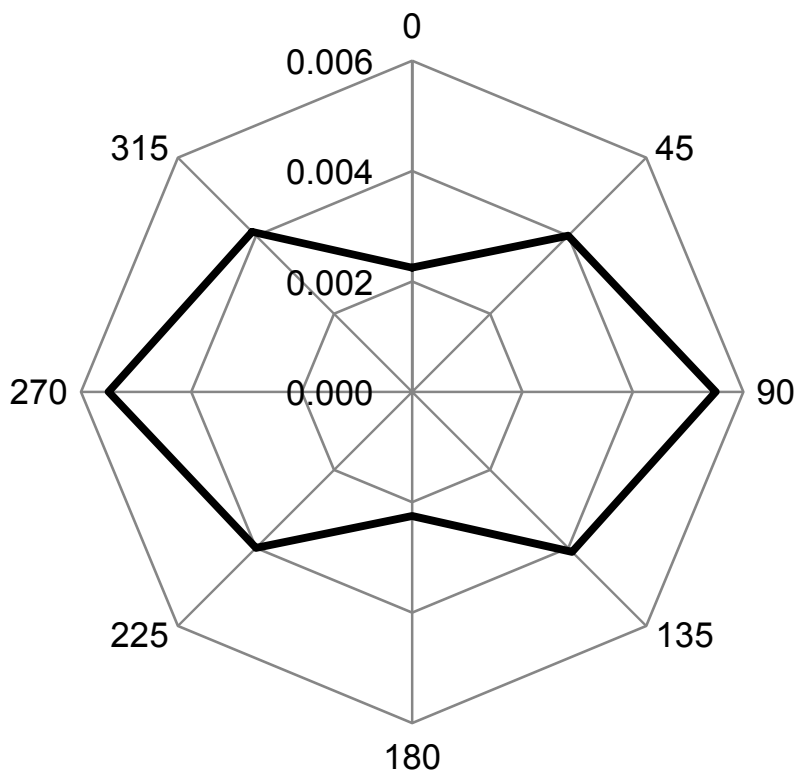

Figure 7: Change in the sill of variograms corresponding to the elevation data of the fracture surface at different directions. Maximum variation is at $90^{\circ}$.

asperity elevation and fluid pressure drop at different directions. The results of the geostatistical analysis are consistent with initial expectations that fluids flowing through a direction with high surface roughness experiences higher pressure drop than fluids flowing through a direction with a relatively smooth surface.

From this study, we can draw the conclusion that geostatistical modelling of fracture surface morphology can be used to predict the hydro-mechanical properties of the fracture. Directions indicated by geostatistical modelling to be highly anisotropic with regards to asperity elevation is expected to cause high pressure drops for any fluids travelling along its surface and conversely, directions with low asperity elevation anisotropy is expected to cause less pressure drops for any fluids travelling along its surface.

\section{Conclusion}

The relationship between a real rock like fracture's morphology and its hydromechanical properties was studied using the geostatistical analysis of the fracture surface asperity elevation data. Variogram analysis was carried out for four different directions at angles $0^{\circ}, 45^{\circ}, 90^{\circ}$ and $135^{\circ}$ measured from the north 


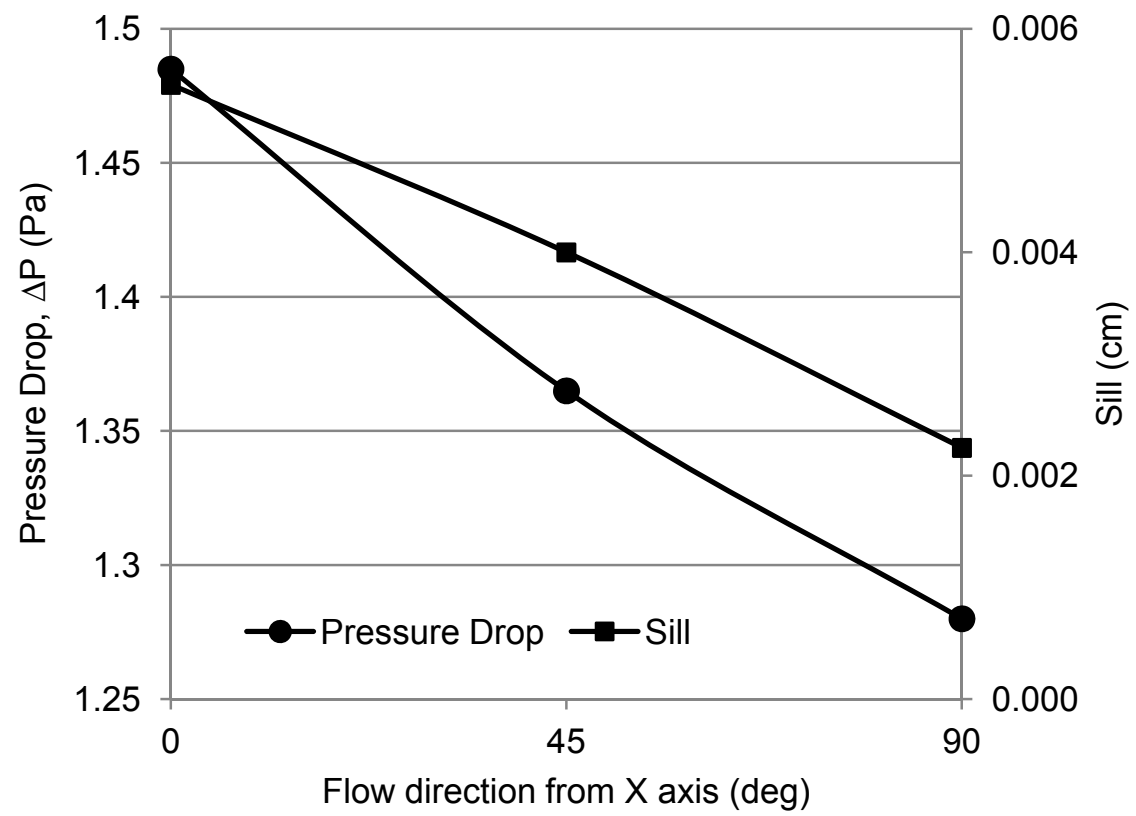

Figure 8: Comparison of fluid flow and geostatistical analysis of the fracture surface.

direction. This allowed for a good representation of surface roughness anisotropy over the whole surface of the mortar sample.

The results of variogram analysis indicated that sample asperity elevations are most directionally dependent in the $0^{\circ}$ direction as the sill value for this direction is substantially lower compared to the other directions modelled. In contrast, the $90^{\circ}$ direction showed to be least directional dependent with a higher sill value than other directions. Comparing these results with the results of the FLUENT fluid flow simulations, a clear correlation between the sill of asperity elevation and fluid pressure drop was observed. A direction along a fracture surface with high value of sill can be indicative of high pressure drops for any fluids that flow along said direction and vice versa.

The results from this study strongly indicate how geostatistical modelling of fracture surface morphology can potentially be used to predict the hydromechanical properties of the fracture. These fractures are common occurrence within petroleum reservoirs and an understanding of how fluid flow is affected by these fractures can play a significant role in real applications such as minimising negative impacts to production of hydrocarbons from the reservoir. 


\section{References}

[1] Witherspoon, P. A., Tsang, Y. W., Long, J. C. S. and Noorishad, J., New approaches to problems of fluid flow in fractured rock masses. U.S. Symposium on Rock Mechanics, pp. 1-15, 1981.

[2] Giacomini, A., Buzzi, O., Ferrero, A. M., Migliazza, M. and Giani, G. P., Numerical study of flow anisotropy within a single natural rock joint. International Journal of Rock Mechanics and Mining Sciences, pp. 1-5. 2008.

[3] Dicman, A., Putra, E. and Schechter, D. S., Modeling Fluid Flow Through Single Fractures Using Experimental, Stochastic and Simulation Approaches. Symposium on Improved Oil Recovery, pp. 6, 2004.

[4] Kamath, J., Lee, S. H., Jensen, C. L., Narr, W. and Wu, H., Modelling fluid flow in complex naturally fractured reservoirs. SPE India Oil and Gas Conference and Exhibition, pp. 1, 1998.

[5] Piggot, A. R. and Elsworth, D., A Hydromechanical Representation of Rock Fractures. U.S. Symposium on Rock Mechanics, pp. 9 - 10. 1991.

[6] Roko, R. O., Daemen, J. J. K. and Myers, D. E., Variogram characterization of joint surface morphology and asperity deformation during shearing. International Journal of Rock Mechanics and Mining Sciences, pp. 1-14. 1997.

[7] Hoerger, S. F. and Young, D. S., Predicting Local Rock Mass Behaviour Using Geostatistics. U.S. Symposium on Rock Mechanics (USRMS), pp. 17. 1987.

[8] Rasouli, V., Personal communication, August 2012, Associate Professor. Petroleum Engineering, Curtin University, Perth, Australia.

[9] Kelkar, M., Perez, G., Spatial Relationships: Estimation and Modelling (Chapter 3). Applied Geostatistics for Reservoir Characterization, pp. 5290. 2002.

[10] Hosseinian, A., Numerical Simulations of Fluid Flow Through a Single Rough Walled Fracture. PhD thesis, Curtin University, 2011.

[11] Rasouli, V., Application of Riemannian multivariate statistics to the analysis of discontinuity surface roughness, $P h D$ thesis, Imperial College, London, 2002. 